\title{
Cortisol and Hippocampal Volume as Predictors of Active Suicidal Behavior in Major Depressive Disorder: Case Report
}

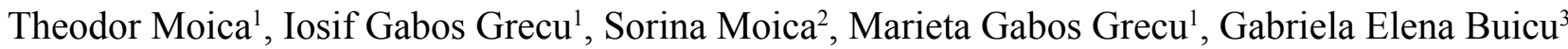 \\ ${ }^{1}$ First Clinic of Psychiatry, University of Medicine and Pharmacy of Tîrgu Mureş, Romania \\ ${ }^{2}$ Department of Industrial Engineering and Management, Petru Maior University of Tîrgu Mureş, Romania \\ ${ }^{3}$ Mental Health Centre, University of Medicine and Pharmacy of Tîrgu Mureş, Romania
}

Background: Suicide is frequently encountered in patients suffering from major depressive disorder (MDD). Since only a third of treated depressed patients are able to achieve remission, in the last few years, new theories have been proposed to better understand the mechanism of this illness. Our paper analyzes the interrelation between cortisol as a marker of neuroendocrine theory as a response to stress, and hippocampal volume subfields in depression as a marker of neurogenesis and neuroplasticity theory.

Case Report: Here we present the case of a 52-yearold male patient with known history of MDD, who died as a result of completed suicide by hanging. The patient had been recently discharged from a psychi- atric clinic, after being hospitalized for a major depressive episode (MDE). The result of the autopsy, medical records, laboratory analysis and a magnetic resonance image (MRI) of the patient were analyzed. Both the right and left volumes of the hippocampus were found to be smaller when compared to normal values reported in the literature. The morning level of cortisol was higher than the normal value.

Conclusion: In a depressed patient with an acute stressful event, high levels of cortisol associated with decreased volume of the hippocampus could represent predictors for an increased risk of suicide.

Keywords: Cortisol, hippocampal volume, suicide
Suicide is considered to be the second leading cause of death among young people and suicidal behavior is frequently encountered in psychiatric patients, especially in major depressive disorder (MDD). The hippocampus is a limbic structure which is involved in the pathophysiological mechanism of MDD, due to its role in cognition, emotions, affect and memory processes. Previous studies have shown hippocampus atrophy and decreased volume, but also the fact that antidepressant medication can induce neurogenesis processes at this level (1).

Recent postmortem studies in depressed patients who committed suicide have shown decreased expression of synapseand glutamate-related genes (2) and altered expression of neuroplasticity-related genes in the hippocampus (3).

Growth factors like brain-derived neurotrophic factor (BDNF) are found in decreased levels in the serum and plasma of patients suffering from MDD. Antidepressant treatment and sleep deprivation therapy were proved to increase serum levels of BDNF (4).

\section{CASE PRESENTATION}

Here we present the case of a 52-year-old male patient with a known history of MDD, who died as a result of completed suicide by hanging from a tree in a forest. An autopsy was performed (Figure 1) at the Legal Medicine Institute and the cause of death was established as asphyxiation due to airway obstruction. Written informed consent was obtained from the legal next of kin of the patient, in this case his wife. 
The patient was a police officer recently discharged from the hospital after being hospitalized for an MDD, single major depressive episode (MDE), severe without psychotic features (according to DSM IV TR). No other medical conditions were identified in this case. Three days after the patient was ad-

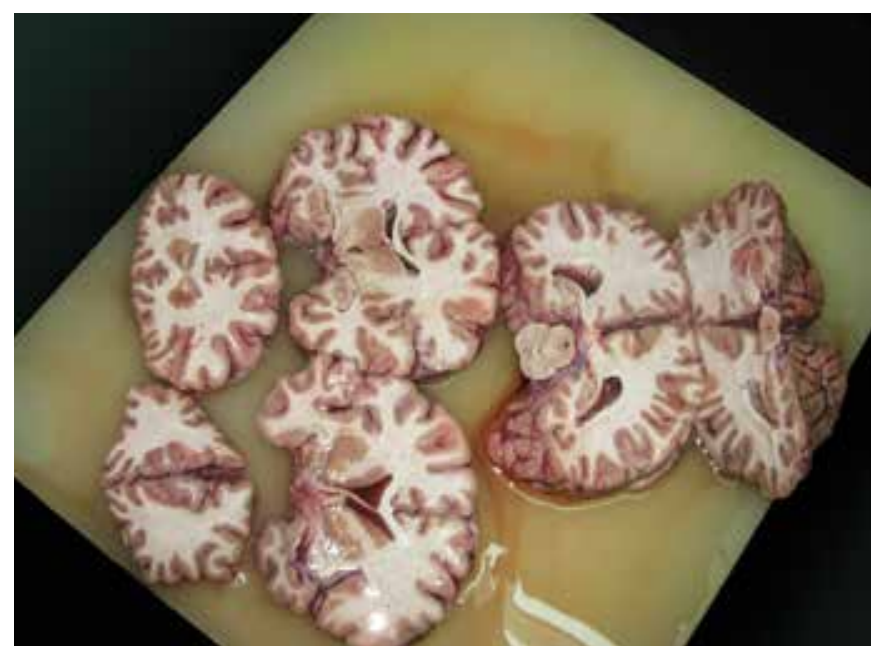

FIG. 1. Sections of the brain - performed at autopsy of the patient.
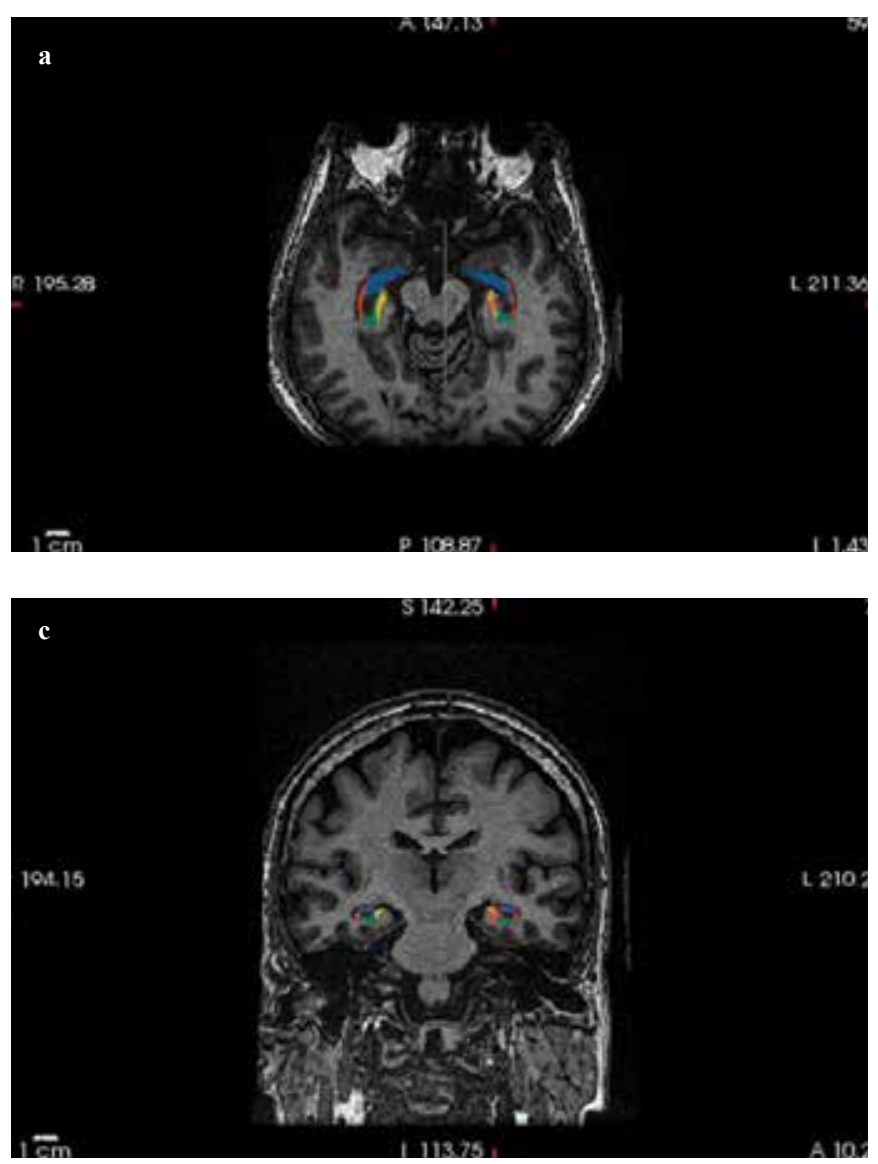

mitted to hospital, lab analyses were performed in a fasting state, including: biochemistry, hematology, lipid panel, urine analysis and one sample of total serum basal morning cortisol level. During hospitalization, no multiple samples of morning cortisol were taken from the blood or saliva of this patient. A magnetic resonance image (MRI) was acquired and no abnormalities were identified. The patient received antidepressant, anxiolytic and hypnotic treatment and he achieved remission of the current MDE after 21 days, with good improvement in his social and professional functionality.

The patient committed suicide 2 weeks after discharge. We found out from his wife that at home he refused to take the antidepressant treatment prescribed by the psychiatrist. He had no previous history of depression and the current MDE was caused by a professional stress factor. The police officer had been informed that in 2 months' time he would not be able to work in the town and he would have to perform his duty in a nearby village, but that this would also be a good opportunity for him to be promoted to a higher position.

A revision of the medical records of the patient was made, including the lab results. On the MRI we performed an automated
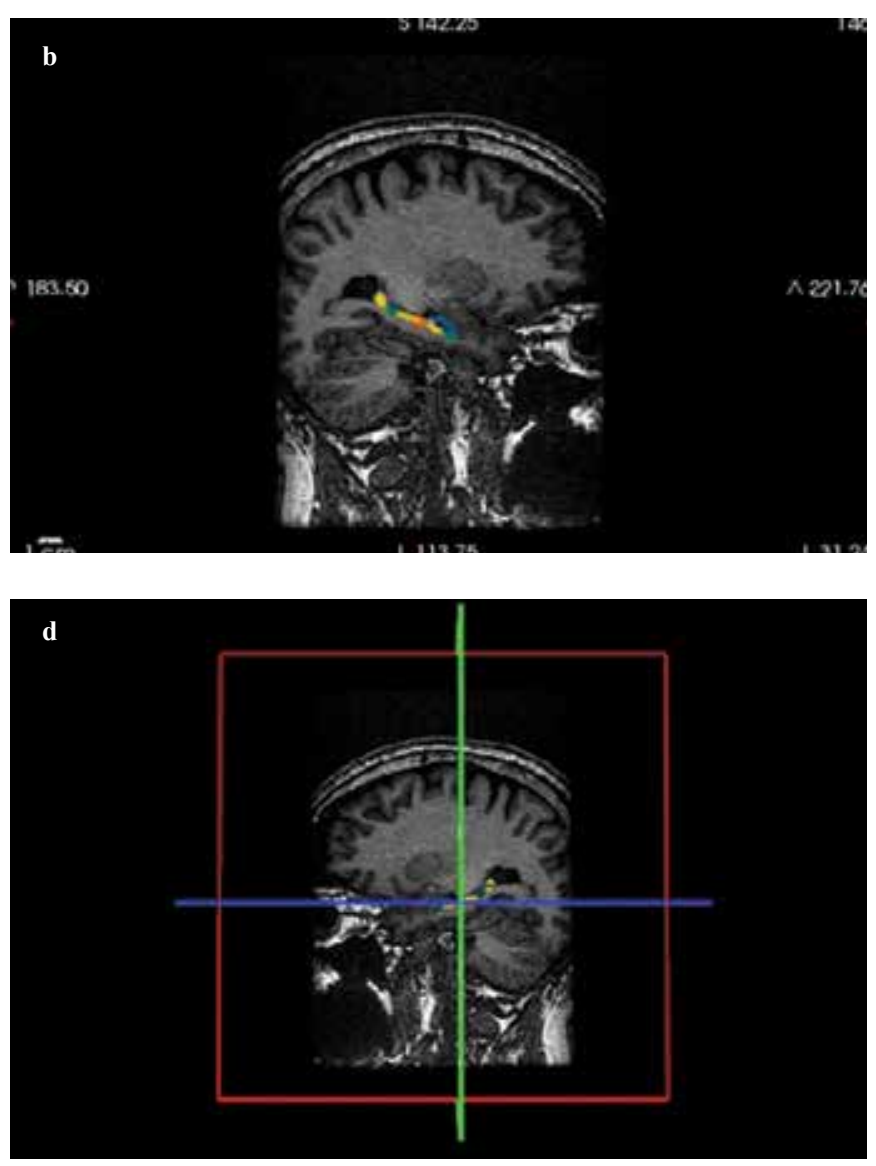

FIG. 2. a-d. Subfield segmentation of the hippocampus with FreeSurfer. Axial (a), sagittal (b) and coronal (c) T1-weighted MR images showing different subfields of the hippocampus. Cross section of the brain (d) - the hippocampus is located below the inferior horn of the lateral ventricle. 
measure of the hippocampal volume and subfield segmentation (Figure 2) with FreeSurfer v.5.3.0 (FreeSurfer, The General Hospital Corp.; Boston MA, USA) on a Linux Station (5).

FreeSurfer is a computer-based program which is able to do an automated measure of cortical and subcortical volumes and surfaces, including the hippocampus. Manual measurement of the hippocampus is complicated and it takes a lot of time and expertise to produce and that was the reason why we chose an automated method of measurement. However, FreeSurfer has some limitations, particularly with respect to newer automated measures recently developed, like Multiple Automatically Generated Templates (MAGeT-Brain), a method that minimizes the number of atlases needed and still achieves similar agreement to multi-atlas approaches. Both the right (3112.3 $\left.\mathrm{mm}^{3}\right)$ and left $\left(2722.82 \mathrm{~mm}^{3}\right)$ volumes of the hippocampus were found to be smaller when compared to normal values reported in the literature (6). The morning level of cortisol was $25.5 \mu \mathrm{g} / \mathrm{dL}$, higher than the A.M. reference value of the laboratory ( 4.3 to $22.4 \mu \mathrm{g} / \mathrm{dL}$ ).

\section{DISCUSSION}

Because only a third of treated depressed patients are able to achieve remission, there is an increased need for identifying biological markers, such as cortisol, that can help us understand the pathophysiological mechanism for each patient and develop more targeted treatments.

Stress is almost always involved in the development of depressive disorders, causing activation of the hypothalamicpituitary-adrenal axis (HPAA), an increase of cortisol, important apoptotic phenomena in the hippocampus and decreased volume of this structure (7).

In a meta-analysis of 12 studies, the volume of the hippocampus has been shown to be significantly decreased in patients with MDD compared with controls, even after they entered remission (8).

In the case we presented, since we had an available MRI dated 4 weeks before the patient committed suicide, we decided to proceed with automated measurement of the hippocampus using FreeSurfer v.5.3.0.

Tae et al. (6) published a comparative study between manual and automated measures (FreeSurfer and IBASPM) of hippocampal volume. We compared our data with the mean for the control group from this study $\left(4017.7 \mathrm{~mm}^{3}\right.$ for the right and $3802.9 \mathrm{~mm}^{3}$ for the left hippocampus). Both the right and the left volumes of the hippocampus were found to be smaller when compared to the normal values reported above.

About $50 \%$ of patients with MDD have hypersecretion of cortisol (9) and the neurotoxic effect of cortisol is higher in the hippocampus and prefrontal cortex, where hypercortisolemia causes apoptosis by decreasing the number of dendritic spines and synapses, reducing the number of glial cells and by causing dendritic atrophy (7). Since the patient had a healthy lifestyle, he had never smoked and he consumed alcohol only occasionally, we were not able to identify other factors that could have had an important neurotoxic impact on the brain. The total basal morning level of cortisol $(25.5 \mu \mathrm{g} / \mathrm{dL})$ was higher than the A.M. reference value reported by the lab.

Coping strategies are methods employed to overcome stress and remain functional. Inadequate control of certain hormones secreted by the hypothalamus can lead to the installation of pathological states like anxiety and depression (10). It seems that these patient coping strategies were maladaptive and this situation might be responsible for a supplementary increase in the level of cortisol and decreased volume of the hippocampus. In the case that we presented, beside the endocrine response to stress and the apoptotic mechanisms that took place in the brain, there were certain features of the personality that influenced the coping mechanisms and might have led to an increased risk of suicide.

We concluded that in a depressed patient with an acute stressing event, high levels of cortisol associated with decreased volume of the hippocampus could represent predictors for an increased risk of suicide. Long-term studies are required to better understand the interrelation between cortisol as a marker of neuroendocrine response to stress, and hippocampal volume subfields in depression as a marker of neurogenesis and neuroplasticity.

\section{Ethics Committee Approval: N/A.}

Informed Consent: Written informed consent was obtained from the spouse of the patient who participated in this study.

Peer-review: Externally peer-reviewed.

Author contributions: Concept - T.M.; Design - T.M.; Supervision - I.G.G., M.G.G.; Resource - T.M., I.G.G., G.E.B; Materials T.M., G.G.İ; Data Collection and/or Processing - T.M., S.M, G.E.B; Analysis and/or Interpretation - T.M., I.G.G, G.E.B, S.M, M.G.G.; Literature Search - T.M., G.E.G, S.M, M.G.G.; Writing - T.M.; Critical Reviews - T.M., I.G.G., G.E.B., S.M., M.G.G.

Acknowledgements: This paper was published under the frame of European Social Found, Human Resources Development Operational Programme 2007-2013, project no. POSDRU/159/1.5/S/136893.

Conflict of Interest: No conflict of interest was declared by the authors.

Financial Disclosure: The authors declared that this study has received no financial support. 


\section{REFERENCES}

1. Serafini G. Neuroplasticity and major depression, the role of modern antidepressant drugs. World J Psychiatry 2012;2:49-57. [CrossRef]

2. Duric V, Banasr M, Stockmeier C, Simen A, Newton SS, Overholser JC, et al. Altered expression of synapse and glutamate related genes in post-mortem hippocampus of depressed subjects. Int $J$ Neuropsychopharmacol 2013;16:69-82. [CrossRef]

3. Fuchsova B, Alvarez Juliá A, Rizavi HS, Frasch AC, Pandey GN. Altered expression of neuroplasticity-related genes in the brain of depressed suicides. Neuroscience 2015;299:1-17.[CrossRef]

4. Gorgulu Y, Caliyurt O. Rapid antidepressant effects of sleep deprivation therapy correlates with serum BDNF changes in major depression. Brain Res Bull 2009;80:158-62. [CrossRef]

5. FreeSurfer (The General Hospital Corporation, Boston MA, USA ("MGH")). FreeSurfer Software 2013 Retrieved May 20 2015, Available from http://surfer.nmr.mgh.harvard.edu/
6. Tae WS, Kim SS, Lee KU, Nam EC, Kim KW. Validation of hippocampal volumes measured using a manual method and two automated methods (FreeSurfer and IBASPM) in chronic major depressive disorder. Neuroradiology 2008;50:569-81. [CrossRef]

7. Walhovd KB, Fjell AM, Reinvang I, Lundervold A, Dale $\mathrm{AM}$, Eilertsen DE, et al. Effects of age on volumes of cortex, white matter and subcortical structures. Neurobiol Aging 2005;26:1261-70. [CrossRef]

8. Maletic V, Robinson M, Oakes T, Iyengar S, Ball SG, Russell J. Neurobiology of depression: an integrated view of key findings. Int J Clin Pract 2007;61:2030-40. [CrossRef]

9. Cowen PJ. Cortisol, serotonin and depression: all stressed out? Br J Psychiatry 2002;180:99-100. [CrossRef]

10. Holsboer F, Ising M. Stress hormone regulation: biological role and translation into therapy. Annu Rev Psychol 2010;61:81-109. [CrossRef] 